\title{
The VuFind based "MT-Katalog" - a customized music library service at the University of Music and Drama Leipzig
}

Anke Hofmann und Dr. Barbara Wiermann

Hochschule für Musik und Theater „Felix Mendelssohn Bartholdy“ Leipzig www.hmt-leipzig.de 
Finc -

Resource Discovery for Saxony

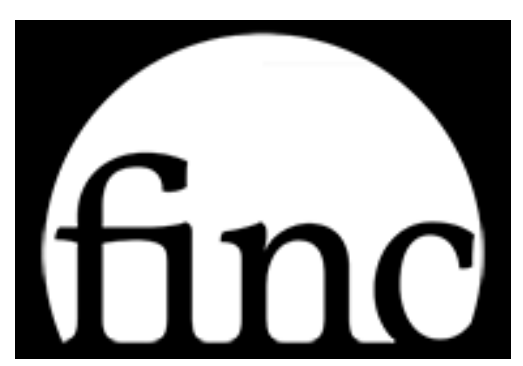

Goal: Installation of Vufind-based Discovery Systems for eleven Saxon University libraries

Project duration: 2011-2014

Responsible body: University Library Leipzig

Financing: European Regional Development Fund (ERDF)

More information: http://blog.finc.info/ 
The VuFind based "MT-Katalog" - a customized music library service at the University of Music and Drama Leipzig

1) finc: general information on software and architecture in the consortium

2) MT-Katalog: Improving search options

3) MT-Katalog: Integrating external resources 


\section{Library of the University of Music and Drama Leipzig}

\section{Physical Collection:}

n Audio-visual material (20.000) n Journals (162)

nicroforms (4.800)

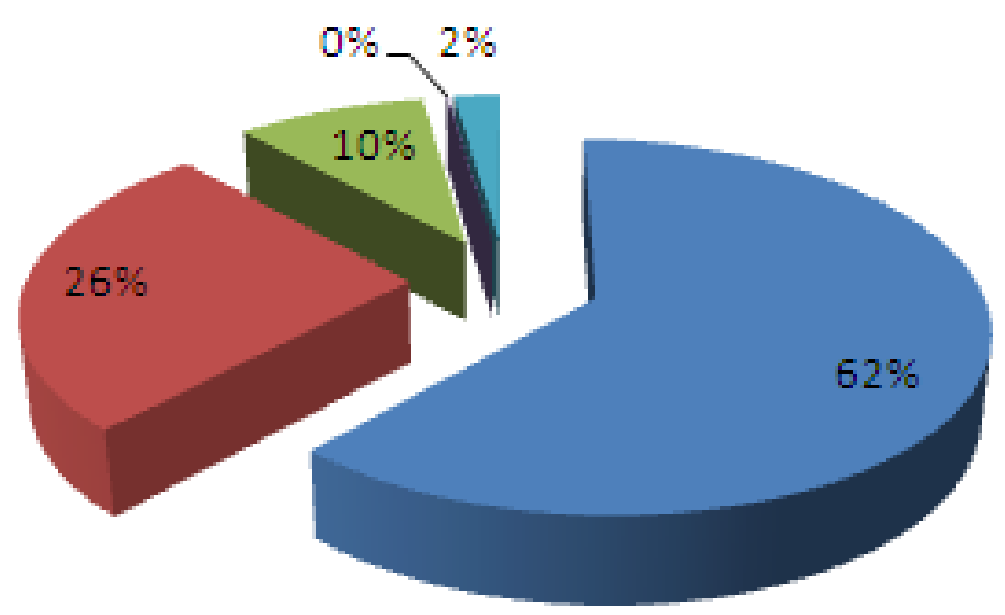

\subsection{Patrons:}

950 music and drama students

350 teachers and professors

http://www.hmt-leipzig.de/bibliothek 


\section{Architecture of the finc Discovery systems}

PrimoCentral Index (selected libraries only)

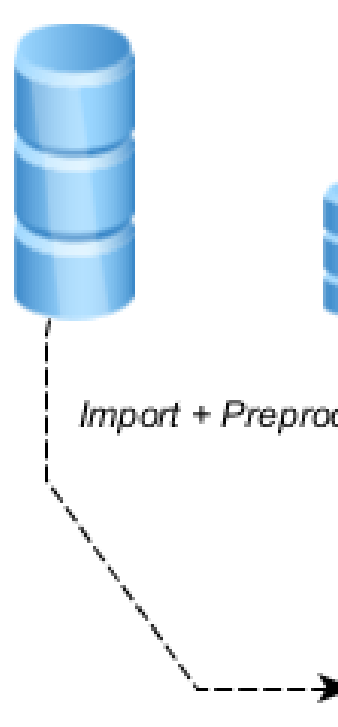

Metadata from the union catalogue (SWB)

(bibliographic data and authority files)
10 other ILS (OPAC)

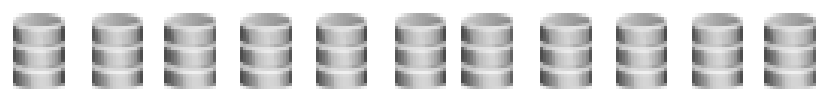

ILS (OPAC)

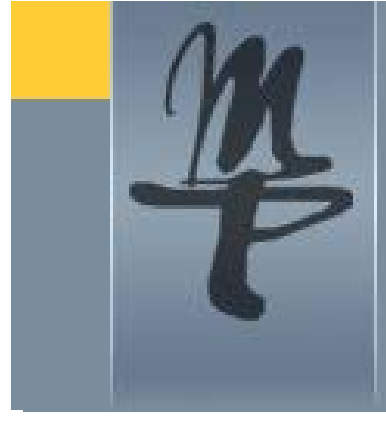

External ressources
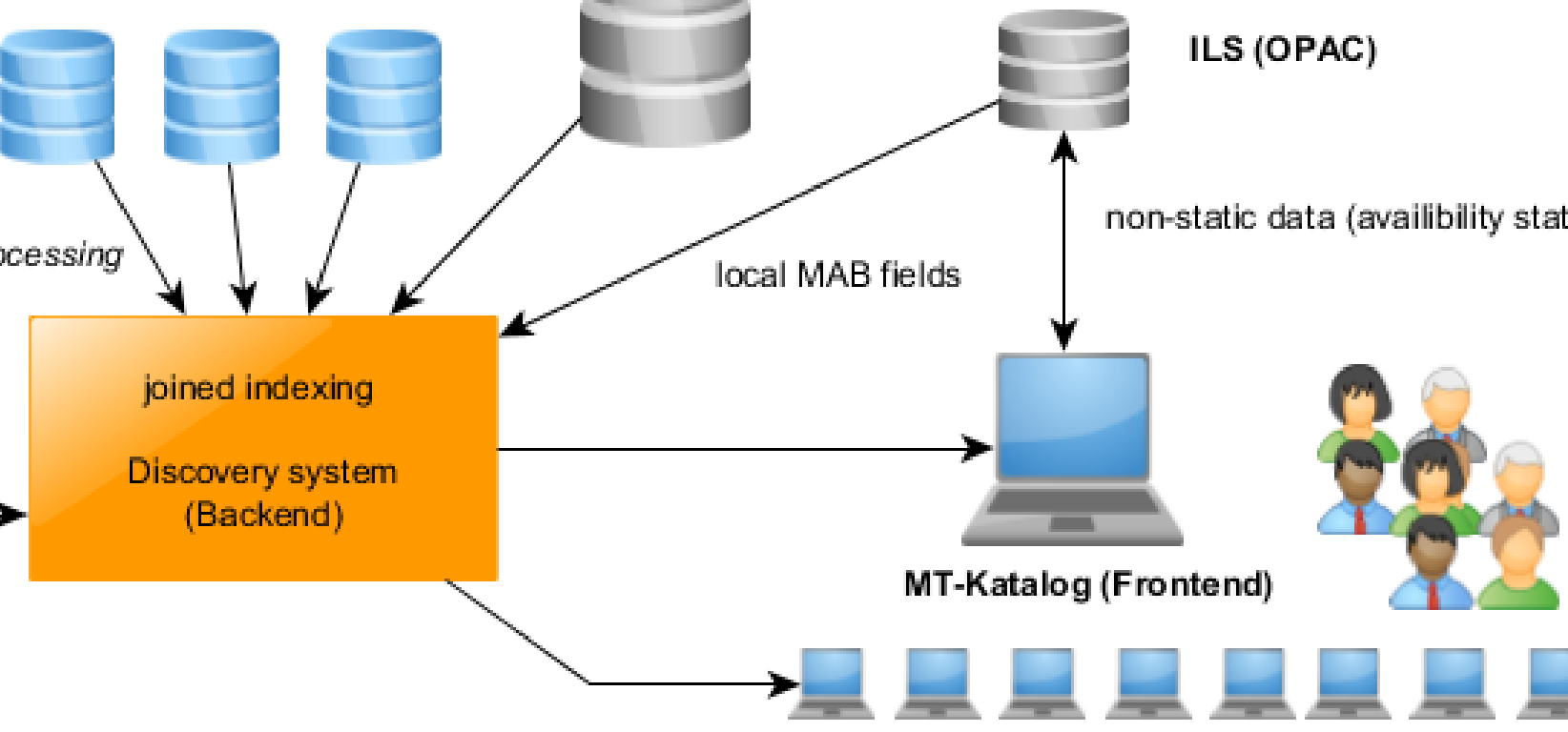

non-static data (availibility status, user account)
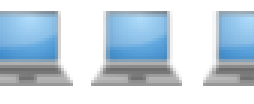

10 other frontends 


Alles Noten Bücher \& mehr Audio Video Zeitschriften
\begin{tabular}{|l|l|l|l|l|l|l}
\hline Starten Sie thre Suche hier... Alle Felder & Suchen
\end{tabular}

Unser Angebot gruppiert nach Medientyp

\begin{tabular}{|c|c|c|c|}
\hline Aufsatz (314) & Buch (47144) & $\mathrm{CD}$ (50912) & CD-ROM (75) \\
\hline DVD (2144) & E-Text (14253) & Gesamttitel (10652) & Handschrift $(52$ \\
\hline Kassette (20) & Medienkombination (3009) & Mikrofilm (112) & Noten (92330) \\
\hline Noten (IMSLP) (45259) & Schallplatte (3032) & Videokassette (560) & Zeitschrift (0055) \\
\hline
\end{tabular}

Unser Angebot gruppiert nach Datensammlung

$\begin{array}{ll}\text { B3Kat (13996) } & \text { Diss online (1529) } \\ \text { Hochschule für Musik "Franz Liszt" } & \text { IMSLP (Petrucci Library) (45289) } \\ \text { Weimar (615) } & \text { Music Online Reference }{ }_{(4058)}\end{array}$

Periodicals Archive Online (528) Qucosa (1110)
Early Music Online (324) Gallica (BNF) (12008)

Lizenzfreie Online-Ressourcen (14504) Lokale HMT-Bestände (119161)

Music Treasures Consortium (1494) Naxos Music Library (39005)

RISM-Digitalisate (8762)

\section{Aktuelles aus der Bibliothek}

Viel $S p a ß$ beim Ausprobieren unseres neuen

MT-Katalogs wünscht das Bibliotheksteam!
Weitere Suchoptionen

Suchverlauf

Suchindex Besetzung/Gattung

Erweiterte Suche
Weitere Angebote der Bibliothek Homepage der HMT-Bibliothek Instrumentenausleihe

Ihr Bestellwunsch

Tel.: 0341.2144633

Mail: bibliothek@hmt-leipzig.de 
Start , Ergebnisliste:

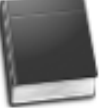

Wahlverwandtschaften: zwei Jahrhunderte musikalischer Wechselwirkun in zwischen Dänemark und Deutschland: [das Buch..

Verfügbar

von Jensen, Anne Ørbaek • veröffentlicht Det Kongelige Bibliotek, København 2004

Signatur: 21092 / Bibliothek Grassistraße

\section{Poetika Kino: Theorie und Praxis des Films im russischen}

\section{Formalismus $=$ Poetika kino}

Beilenhoff, Wolfgang • veröffentlicht Suhrkamp, Frankfurt 2005

Favoriten if Verfügbar

Signatur: 21923 / Bibliothek Grassistraße

Wem gehört Musik?: Warschau und Wilna im Widerstreit nationaler und städtischer Musikkulturen vor 1939

von Ritter, Radiger • veröffentlicht Steiner, Stuttgart 2004

Verfügbar

Signatur: 21283 / Bibliothek Grassistraße

Jazz im Totalitarismus: eine komparative Analyse des politisch motivierten Umgangs mit dem Jazz während der Zeit...

von Lacke, Martin • veröffentlicht LIT, Manster 2004

Verfügbar

Signatur: 22658 / Bibliothek Grassistraße

New edition of the complete works, 22b, Arrangements of works by

Favoriten other composers (II) / ed. by lan Rumbold

Berlioz, Hector • Rumbold, lan • veröffentlicht Barenreiter, Kassel ; Basel 2004

\section{Suche einschränken}

- Zugang

$\square$ Online-Ressourcen (136328)

$\square$ Lokale HMT-Bestände (131557)

- Medientyp

$\square$ Noten (92830)

$\square \mathrm{CD}(50912)$

$\square$ Buch (47144)

$\square$ Noten (IMSLP) (45269)

$\square$ E-Text (14253)

mehr.

- Datensammlung

$\square$ Lokale HMT-Bestände (119161)

$\square$ IMSLP (Petrucci Library) (45269)

$\square$ Naxos Music Library (38906)

$\square$ Lizenzfreie Online-Ressourcen (14804)

$\square$ B3Kat (13936)

mehr...

- musikalische Ausgabeform

$\square$ Quelle (26267)

$\square$ Partitur (20177)

$\square$ Stimmen (8765)

$\square$ Klavierauszug (4512)

$\square$ Aufführungsmaterial (2246) 


\section{Facets of the MT-Katalog}

\section{- Zugang}

$\square$ Online-Ressourcen (134793)

$\square$ Lokale HMT-Bestände (131448)

- Medientyp

$\square$ Noten (92810)

$\square \mathrm{CD}(50900)$

$\square$ Buch (47090)

$\square$ Noten (IMSLP) (45269)

$\square$ E-Text (12721)

mehr ...

\section{- Datensammlung}

$\square$ Lokale HMT-Bestände (119052)

$\square$ IMSLP (Petrucci Library) (45269)

$\square$ Naxos Music Library (38906)

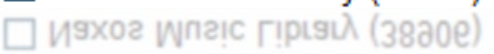

- musikalische Ausgabeform

$\square$ Quelle (26259)

$\square$ Partitur (20163)

$\square$ Stimmen (8760)

$\square$ Klavierauszug (4510)

$\square$ Aufführungsmaterial (2246)

mehr ...

- musikalische Besetzung

$\square$ Oper (2169)

$\square$ Eins Klavier Solo (2021)

$\square$ Orchester Sinfonie (1286)

$\square$ Vier Violine (2), Viola, Violoncello

Quartett (1249)

$\square$ Eins Orgel Solo (818)

mehr...

wөpt.

$\square$ Elua oเฮิเ ว이 (8.8)

\section{- Filmgenre}

$\square$ Musikaufzeichnung (27)

$\square$ Operninszenierung (16)

$\square$ Filmporträt (15)

$\square$ Filmbericht (13)

$\square$ Konzertaufführung (11) mehr ...

- Person / Institution

$\square$ Haydn, Joseph 1732 - 1809 (376)

$\square$ Mozart, Wolfgang Amadeus 1756 1791 (334)

$\square$ Beethoven, Ludwig van $1770-1827$ (329)

$\square$ Rundfunk-Sinfonieorchester (247)

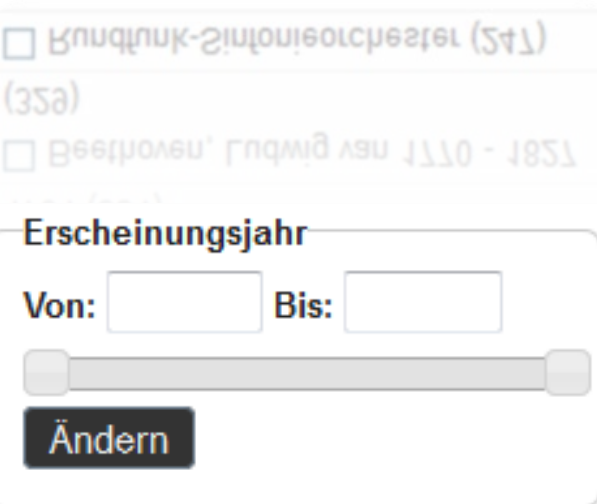




\section{Optimized Search options Facet Film genre - MARC 937}

\begin{tabular}{|c|c|c|c|}
\hline vertugba & \multicolumn{2}{|l|}{ Signatur: DVD 11789 / Bibliothek Grassistraße-Magazin } & $\begin{array}{l}\text { - Filmgenre } \\
\square \text { Spielfilm (791) }\end{array}$ \\
\hline \multirow{6}{*}{ Verfügbar } & Nosferatu & \multirow{6}{*}{ Favoriten } & $\square$ operninszenierung (284) \\
\hline & \multirow{4}{*}{$\begin{array}{l}\text { Murnau, Friedrich Wilhelm • Schreck, Max • Wangenheim, Gustav von ... • veröffentlicht } \\
\text { Eureka Video, [S.I.] } 2000\end{array}$} & & $\square$ Literaturverfilmung (271) \\
\hline & & & $\square$ Dokumentarfilm (181) \\
\hline & & & $\square$ Musikaufzeichnung (181) \\
\hline & & & $\square$ Fernsehserie (153) \\
\hline & Signatur: DVD 11234 / Bibliothek Grassistraße-Magazin & & $\square$ Theaterinszenierung (123) \\
\hline & The Criterion collection & \multirow{4}{*}{ Favoriten $\hat{\imath}$} & $\square$ Filmporträt (99) \\
\hline & veröffentlicht The Criterion Collection, [S.I.] & & $\square$ Kompilation (92) \\
\hline & & & $\square$ Opernaufführung (87) \\
\hline & & & $\square$ Ballettinszenierung (68) \\
\hline & \multirow[b]{2}{*}{ [Gesamttitel DVD] } & \multirow{2}{*}{ Mehr $\mathbb{V}$} & $\square$ Lehrfilm (68) \\
\hline & & & $\square$ Stummfilm (68) \\
\hline & \multirow{4}{*}{$\begin{array}{l}\text { Roman Polanskis Verfilmung von Macbeth : ein Herrscher wird } \\
\text { gejagt } \\
\text { Polanski, Roman • Shakespeare, William • Finch, John ... • veröffentlicht Columbia } \\
\text { Tristar Home Entertainment, [München] } 2002\end{array}$} & \multirow[t]{6}{*}{ Favoriten 党 } & $\square$ Theateraufführung (67) \\
\hline & & & $\square$ Lernprogramm (58) \\
\hline & & & $\square$ Kurzfilm (50) \\
\hline Ver & & & $\square$ Filmbericht (49) \\
\hline & \multirow{2}{*}{ Signatur: DVD 11170 / Bibliothek Grassistraße-Magazin } & & $\square$ Fernsehfilm (31) \\
\hline & & & $\square$ Musikfilm (31) \\
\hline & \multirow{4}{*}{$\begin{array}{l}\text { Alcina : dramma per musica in tre atti (1735); from the Staatsoper } \\
\text { Stuttgart } 1999 \\
\text { von Händel, Georg Friedrich • veröffentlicht Arthaus Musik [u.a.], [München] } 2000\end{array}$} & \multirow[t]{6}{*}{ Favoriten \{} & $\square$ Musicalinszenierung (29) \\
\hline & & & $\square$ Konzertaufführung (24) \\
\hline & & & $\square$ Opernverfilmung (20) \\
\hline Verfügbar & & & $\square$ Trickfilm (17) \\
\hline & \multirow{2}{*}{ Signatur: DVD 11509 / Bibliothek Grassistraße-Magazin } & & $\square$ Fernsehinszenierung (16) \\
\hline & & & $\square$ Tanzfilm (15) \\
\hline \multirow{2}{*}{ pats } & \multirow{2}{*}{$\begin{array}{l}\text { Le nozze di Figaro : KV 492; opera buffa in quattro atti = The } \\
\text { marriage of Figaro = Die Hochzeit des Figaro }\end{array}$} & \multirow[t]{2}{*}{ Favoriten } & $\square$ Filmdokumentation (14) \\
\hline & & & $\square$ Operetteninszenierung (13) \\
\hline
\end{tabular}




\section{Musical Presentation}

Terms for „piano reduction“ in MAB 359 and 403

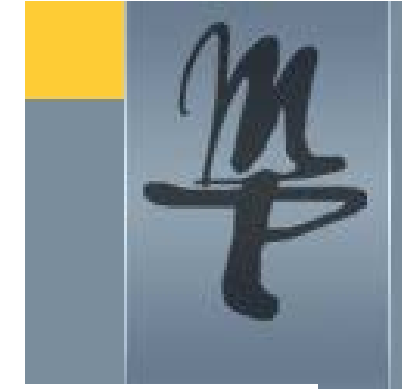

ర্ّ

piano vocal score

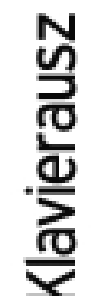

Piano Reductionpiano Wyciag fortpianowy deutscher KlavierauszûgIm Klavierauszuge mit GesangVocal score complete vocal selectionšKlavier-Ausz Klavírny výtah upravil skladatel

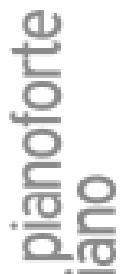
Revue doigtée et éditée avec accompagnement de piano
piano Partition avec réduction pour piano Piano-vocal score 등 Arranged for piano Perelozenie dlja penija s fortepiano Nㅡㄹ Ausg. f. KlPereloenie dlja penija s fortepiano

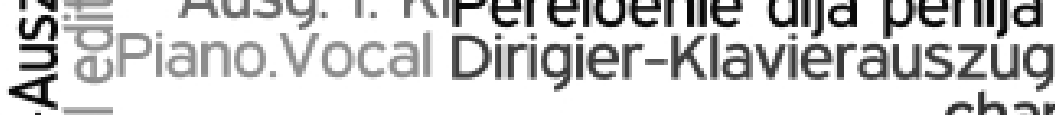
chantlavierauzug

产 Klav.-Ausz

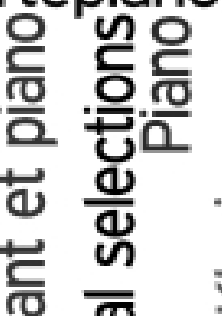

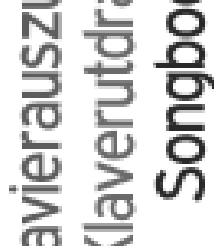
$\frac{5}{5}$

$\frac{\pi}{0}$

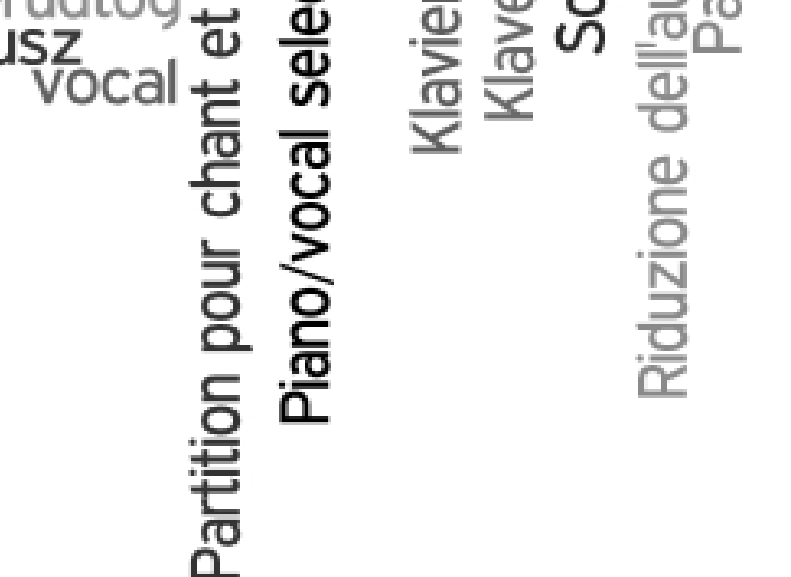




\section{Five musical presentations used in the MT-Katalog}

- Aufführungsmaterial

- Partitur

- Stimmen

- Klavierauszug

- Quelle performance material

full score

parts

piano reduction

copies of primary facsimiles,

source materials microforms, digitized materials music prints with more than 9 parts 


\section{Musical presentations used in the MT-Katalog}

\section{Violinkonzert D-dur, op. 61}

von Beethoven, Ludwig van • veröffentlicht Breitkopf \& Härtel, Wiesbaden [u.a.] c 1990

Aufführungsmaterial

Verfügbar

Mehrere Exemplare / Bibliothek Grassistraße-Magazin

\section{Konzert für Violine und Orchester D-dur, op. 61}

von Beethoven, Ludwig van • veröffentlicht Breitkopf \& Härtel, Wiesbaden [u.a.] c 1973

\section{Partitur}

Verfügbar

Signatur: M.pr.IV $7052 \mathrm{P}$ / Bibliothek Grassistraße-Magazin

Violin-Konzert D-Dur ... für Violine und Orchester op....

von Beethoven, Ludwig van • veröffentlicht Schott, Mainz [u.a.] c 1971

Partitur · Stimmen

Verfügbar

Mehrere Exemplare / Bibliothek Grassistraße

\section{Violin-Concert op. 61}

von Beethoven, Ludwig van • veröffentlicht Peters, Leipzig [0.J.]

Klavierauszug

Vormerkbar
Favoriten 1

Favoriten $\hat{A}$

Suche einschränken

- Zugang

$\square$ Lokale HMT-Bestände (16)

- Medientyp

$\square$ Noten

$\square$ Schallplatte (18)

$\square \mathrm{CD}(8)$

$\square$ Buch (1)

$\square$ E-Text (1)

mehr...

- Datensammlung

$\square$ Lokale HMT-Bestände (16)

- musikalische Ausgabeform

$\square$ Partitur (6)

$\square$ Klavierauszug (3)

$\square$ Stimmen (3)

$\square$ Aufführungsmaterial (2)

- musikalische Besetzung

$\square$ Violine, Orchester Konzert (5)

$\square$ Zwei Violine, Klavier Duo, Konzert (3) 
Mapping to get the relevant information for MAB 951 / MARC 970 musical representation

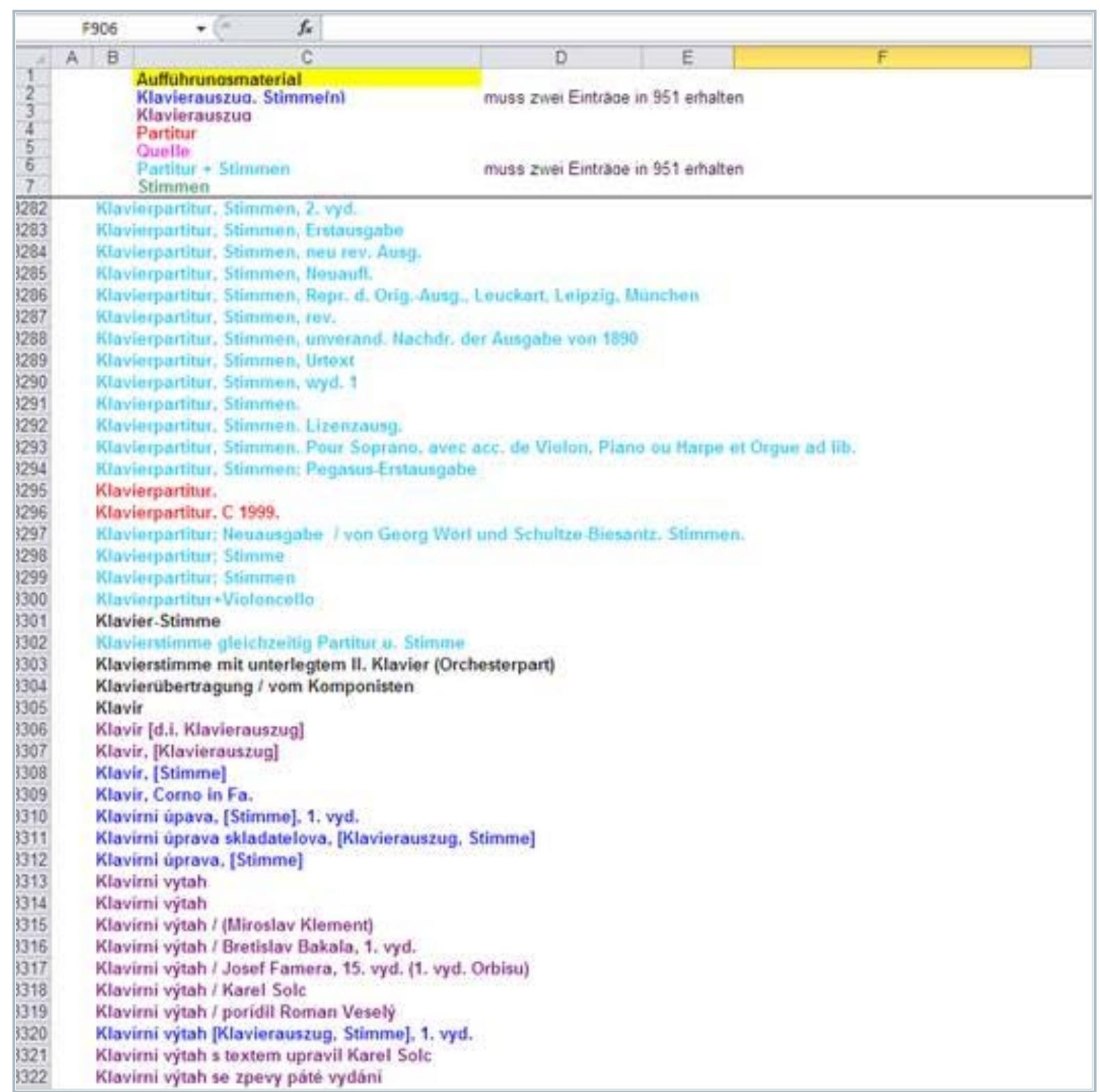




\section{Musical presentations used in the MT-Katalog}

\section{Violinkonzert D-dur, op. 61}

von Beethoven, Ludwig van • veröffentlicht Breitkopf \& Härtel, Wiesbaden [u.a.] c 1990

Aufführungsmaterial

Verfügbar

Mehrere Exemplare / Bibliothek Grassistraße-Magazin

\section{Konzert für Violine und Orchester D-dur, op. 61}

von Beethoven, Ludwig van • veröffentlicht Breitkopf \& Härtel, Wiesbaden [u.a.] c 1973

\section{Partitur}

Verfügbar

Signatur: M.pr.IV $7052 \mathrm{P}$ / Bibliothek Grassistraße-Magazin

Violin-Konzert D-Dur ... für Violine und Orchester op....

von Beethoven, Ludwig van • veröffentlicht Schott, Mainz [u.a.] c 1971

Partitur · Stimmen

Verfügbar

Mehrere Exemplare / Bibliothek Grassistraße

\section{Violin-Concert op. 61}

von Beethoven, Ludwig van • veröffentlicht Peters, Leipzig [0.J.]

Klavierauszug

Vormerkbar
Favoriten 1

Favoriten $\hat{A}$

Suche einschränken

- Zugang

$\square$ Lokale HMT-Bestände (16)

- Medientyp

$\square$ Noten

$\square$ Schallplatte (18)

$\square \mathrm{CD}(8)$

$\square$ Buch (1)

$\square$ E-Text (1)

mehr...

- Datensammlung

$\square$ Lokale HMT-Bestände (16)

- musikalische Ausgabeform

$\square$ Partitur (6)

$\square$ Klavierauszug (3)

$\square$ Stimmen (3)

$\square$ Aufführungsmaterial (2)

- musikalische Besetzung

$\square$ Violine, Orchester Konzert (5)

$\square$ Zwei Violine, Klavier Duo, Konzert (3) 


\section{Verbal representation of the classification system RVK}

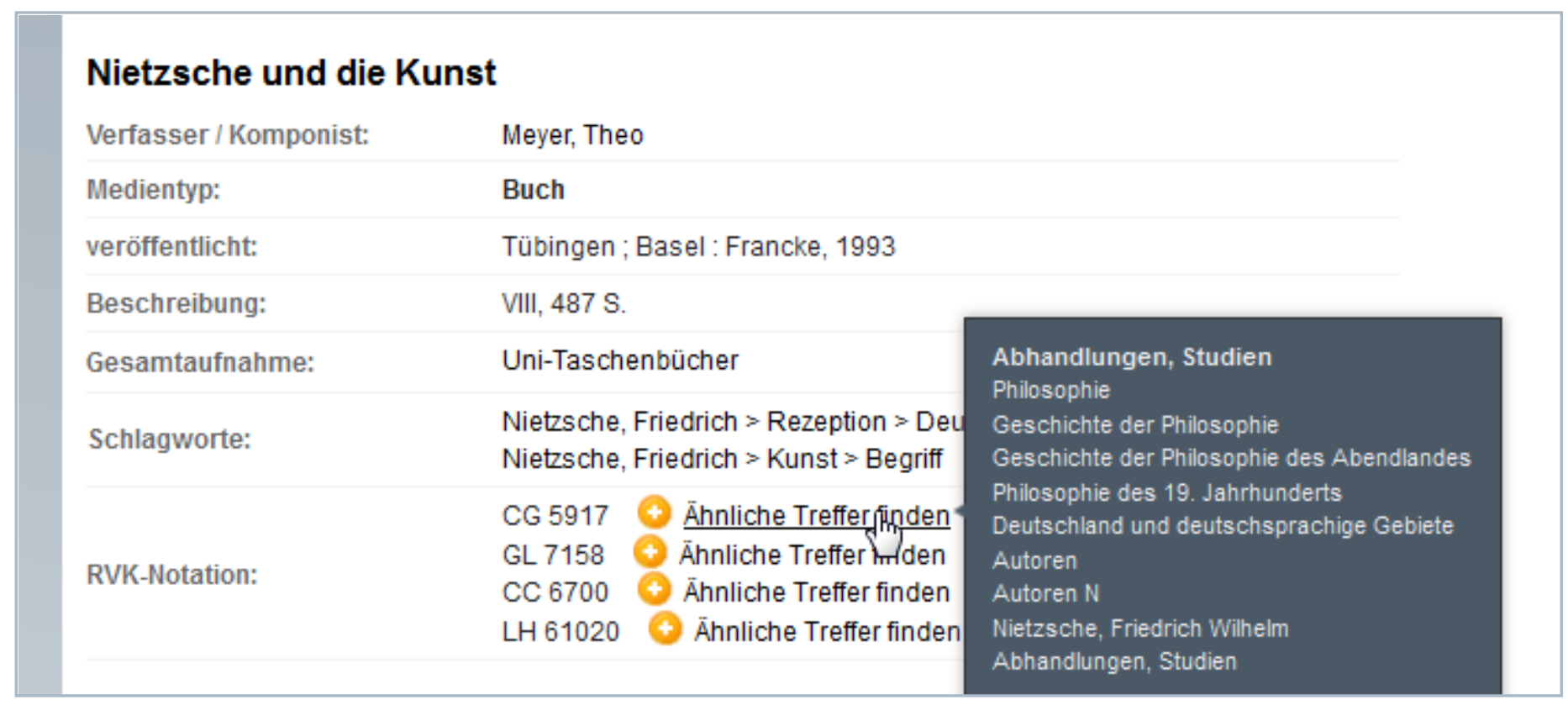

Kunsttheorie und Geschichte der Kunsttheorie Kunstgeschichte

Allgemeines. Allgemeine Kunstgeschichte

Theorie und Praxis der Bildenden Kunst

Theorie der Bildenden Kunst

Kunsttheorie und Geschichte der Kunsttheorie
Geschichte der Ästhetik

Philosophie

Systematische Philosophie

Ästhetik und Kunstphilosophie

Geschichte der Ästhetik
Sekundärliteratur.

Germanistik. Niederlandistik. Skandinavistik

Deutsche Literatur

Das 19. Jahrhundert

Einzelne Autoren

Autoren N

Nietzsche, Friedrich

Sekundärliteratur. 


\section{External resources of the MT-Katalog I}

- Licensed materials

(e. g. Naxos Music library, Music online reference)

- Resources of library projects as

- Data from open access repositories (e. g. Diss online, Qucosa, SWB/LFER)

- Data from digitization projects of different libraries (e. g. Music Treasures Consortium/LoC, Gallica/BNF, Digitale Sammlungen der BSB, Early Music online/BL)

- Data from the most famous Music Wiki: International Music library project (www.imslp.org) 


\section{External resources of the MT-Katalog II}

- licensed materials: e-books, e-papers and e-scores (5.500 titles)

q digital born open access publications 11.200 titles)

retro digitized materials (38.000 titles)

- licensed audio files (39.000 titles)

- IMSLP Wiki data (45.000 works)

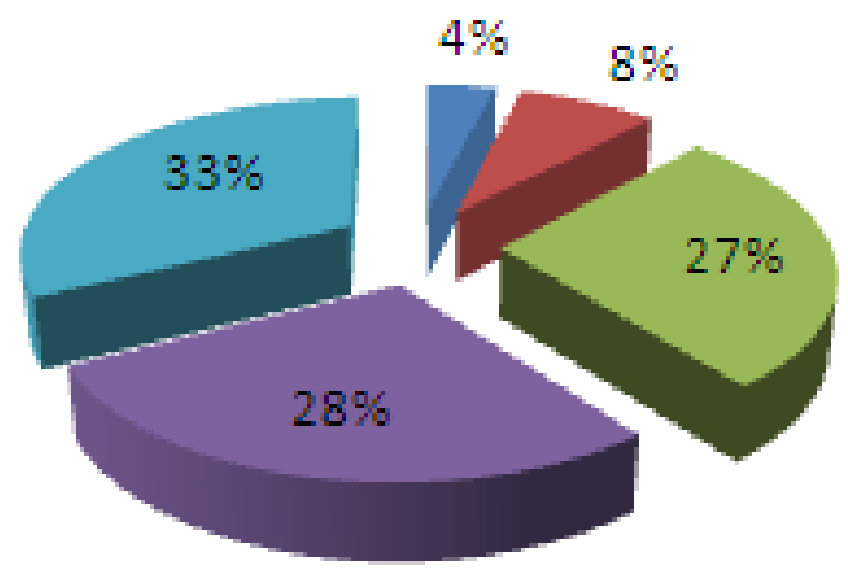




\section{External Resources of the MT-Katalog III}

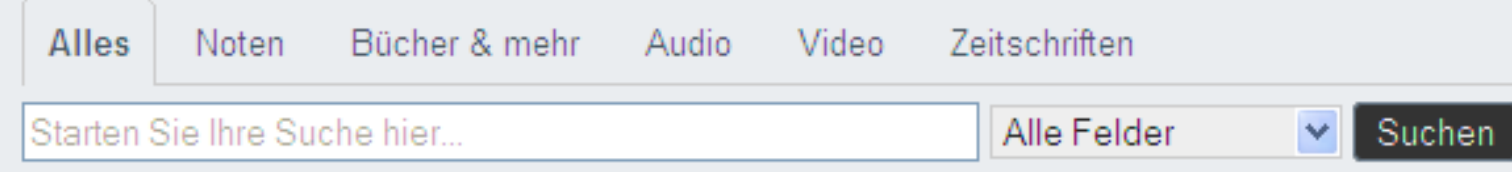

Erweiterte Suche $\cdot$ Suchindex Besetzung/Gattung

Unser Angebot gruppiert nach Medientyp

$\begin{array}{llll}\text { Aufsatz (314) } & \text { Buch (47144) } & \text { CD (50912) } & \text { CD-ROM (75) } \\ \text { DVD (2144) } & \text { E-Text (14253) } & \text { Gesamttitel (10652) } & \text { Handschrift (52) } \\ \text { Kassette }(29) & \text { Medienkombination (3209) } & \text { Mikrofilm (112) } & \text { Noten (92850) } \\ \text { Noten (IMSLP) (45259) } & \text { Schallplatte (3052) } & \text { Videokassette (560) } & \text { Zeitschrift (8055) }\end{array}$

nicht zugeordnet (15)

Unser Angebot gruppiert nach Datensammlung

B3Kat (13996) Diss online (1529)

Hochschule für Musik "Franz Liszt" IMSLP (Petrucci Library) (45269)

Weimar (615)

Periodicals Archive Online (528)
Music Online Reference (4058)

Qucosa (1110)
Early Music Online (324)

Gallica (BNF) (12008)

Lizenzfreie Online-Ressourcen (14504) Lokale HMT-Bestände (119161)

Music Treasures Consortium (1494) Naxos Music Library (39906)

RISM-Digitalisate (8762) 


\section{Selection criteria for external resources}

- Diss online / German national library

- Digital collection of the Bavarian State library

- Gallica / Bibliothèque nationale de France

- LFER / SWB Konstanz Media type and classification

- Dart: Europe E-theses ???

Portal / Association of European Research Libraries
DDC: Music (780), Film (791), Drama, Dance (792), Pedagogy (370)

SSG-Indication: 9.2 musicology

Separate OAI-set for music scores provided by the BNF 


\section{Lizenzfreie Online- \\ Ressourcen \\ im SWB}

\section{Selection criteria for the LFER data}

- Media type

- DDC

- Old classification system of the National library

- RVK notation

PICA $1140=$ MARC 935 \$c = muno

OR

PICA $1140=$ MARC 935 \$c = muto

OR

PICA $5010=$ MARC 0820 \$a = (780 OR 791 OR 792 OR 020 OR 370)

OR

PICA 5051 = MARC 0820 \$a = (780 OR 791 OR 792 OR 020 OR 370)

OR

PICA $5050=$ MARC $084 \$ a=(02$ OR 48 OR 49 OR 22)

OR

PICA 5090 = MARC 084 \$a / MARC 936 rv\$a = (LP* OR LQ* OR LR* OR LS* OR C* OR D*)

The number of about 250.000 data sets like this was narrowed to 14.000 data sets.dealing with music, drama, pedagogy 
General preprocessing of data sets from external resources

\section{Media types for external resources (Preprocessing)}

electronic texts

OD

digitized music scores

DN

digitized music scores from IMSLP

PN

online audio files

ECD

\section{- Zugang}

$\square$ Online-Ressourcen (134799)

$\square$ Lokale HMT-Bestände (131476)

- Medientyp

$\square$ Noten (92813)

$\square$ CD (50906)

$\square$ Buch (47109)

$\square$ Noten (IMSLP) (45269)

$\square$ E-Text (12724)

$\square$ Gesamttitel (10622)

$\square$ Zeitschrift (8055)

$\square$ Medienkombination (3205)

$\square$ Schallplatte (3032)

$\square$ DVD (2142)

\section{Media types} for the physical collection scores

$\mathrm{N}$

monographs B

serials

Z

CDs

CD 


\section{Electronic resources}

seperating different media types (Music scores and E-texts)

Selection criteria:

Edition Statement (MARC 250):

Partitur

Klavierauszug

Stimmen
- Zugang

$\square$ Online-Ressourcen (13936)

- Medientyp

$\square$ E-Text (8310)

$\square$ Noten (5626)

- Datensammlung

B BKat

- musikalische Ausgabeform

$\square$ Quelle (4118)

Electronic location and access (MARC 856) including the call numbers of the physical items

“Mus.pr." OR „LB Coburg“ OR „Liturg“ OR „Hbm/G*“

OR „Hbm/D*" OR „rar." OR „St.th.“ OR „Mus.ms.“ OR „Mus.coll“ OR „Mus.N.“ 


\section{Example of a data set from the Bavarian state library catalog in the MT-Katalog}

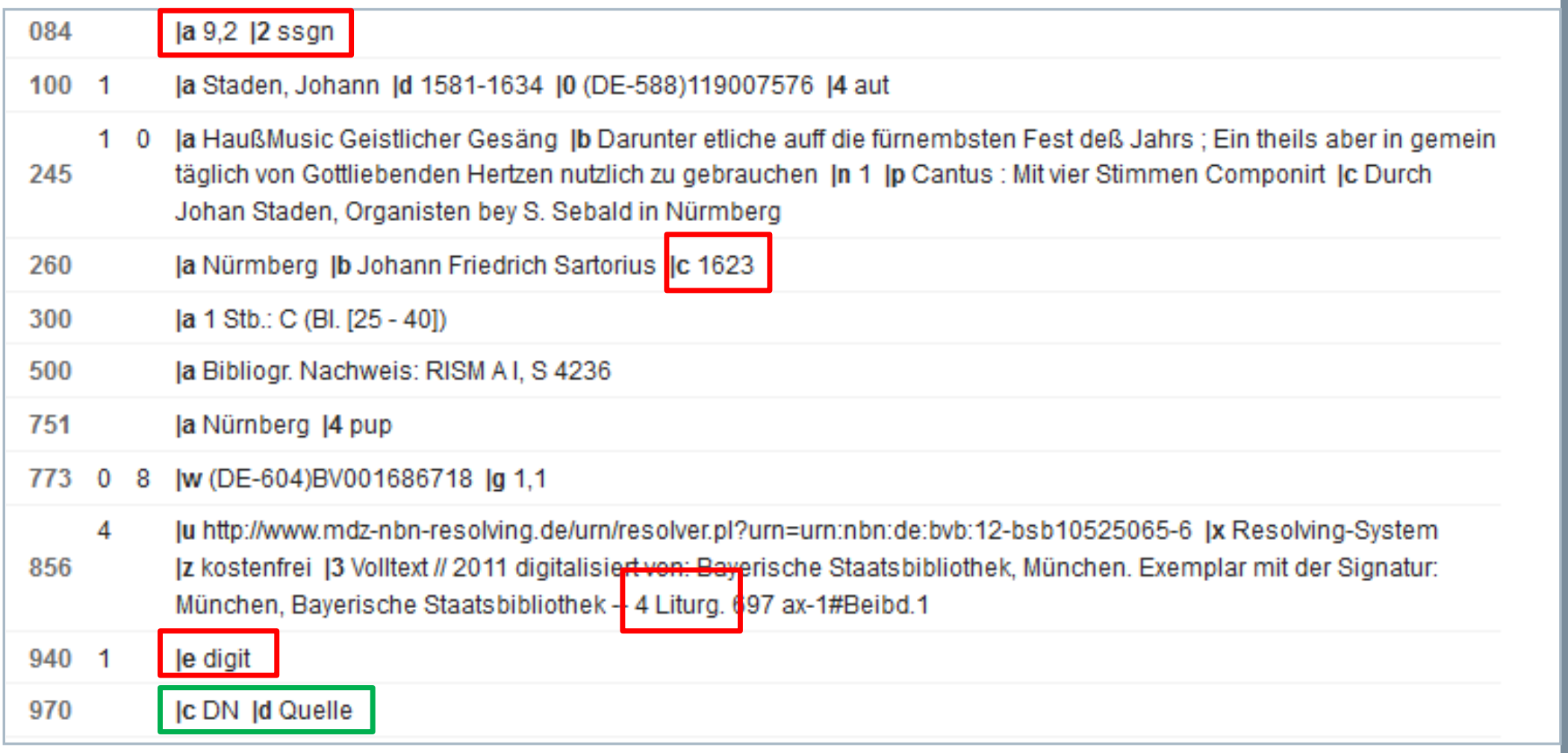




\section{Search in the MT-Katalog for the composer „Kuhnau“}

In addition to the result list for library holdings and external resources the user gets references of two major databases

Choral Music (Sacred) - SWEELINCK, J.P. I KUHNAU, J. I LOTTI, A. I ECCARD, J. I DURANTE, F...

Anerio, Felice $\cdot$ Bach, Johann Michael $\cdot$ Durante, Francesco ... • veröffentlicht Naxos Digital Services Ltd, Hong Kong [2009]

[CD online]

II Combattimento trà David e Goliath: = Der Streit zwischen David und Goliath

von Kuhnau, Johann • veröffentlicht Deutsche Grammophon, [Hamburg] [19]55

Verfügbar

Signatur: SP 468 / Bibliothek Grassistraße-Magazin

Zwei Begräbnismotetten: für fünfstimmigen Chor a cappella

von Kuhnau, Johann • veröffentlicht Carus-Verl, Stuttgart c 1998

Aufführungsmaterial

Favoriten 
For more information see http://katalog.hmt-leipzig.de/

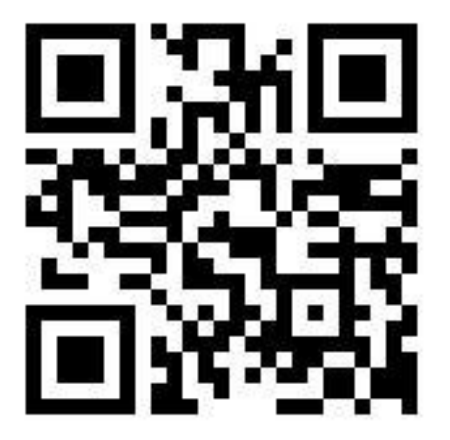

Or visit our blog at http://bibblog.hmt-leipzig.de/

Contact information:

anke.hofmann@hmt-leipzig.de

barbara.wiermann@hmt-leipzig.de 